MATEC Web of Conferences 22,02005 (2015)

DOI: $10.1051 /$ matec conf/ 20152202005

(C) Owned by the authors, published by EDP Sciences, 2015

\title{
Implementation of Pulse Radar Waveform Based on Software Radio Platform
}

Dong Wang, Jian Dong \& Shunping Xiao

School of Electronic Science and Engineering, National University of Defense Technology, Changsha, Hunan, China

ABSTRACT: Based on the frequency and phase modulated signal, the authors design some commonly-used pulse radar baseband waveform, such as linear frequency modulated waveform, nonlinear frequency modulated waveform, Costas waveform, Barker coding waveform and multi-phase coded waveform, and the authors compare their performance, such as the peak side lobe ratio, the Rayleigh resolution in time and distance resolution. Then, based on the software radio platform NI PXIe-5644R, the authors design the timing control procedures and waveform launch procedures, and make the sampling rate, center frequency, pulse period and signal power flexible and controllable. After that, the authors test and verify the various waveforms.

Keywords: signal modulation; radar baseband waveform; software radio; timing control

\section{INTRODUCTION}

Software radio technology arose during 70 80s in the twentieth century, and had been first proposed by Jeseph Mitola at a meeting of the American communication system in 1992 May[1]. Its core idea is that the hardware function can be realized by software programming in an open and standard hardware platform. And it emphasizes the openness and full programmability of the system structure, which means that the configuration structure of hardware can be changed through the software updating, so that the hardware can be upgraded and realize some new functions[2].

Radar is electronic equipment which uses the radio wave to discover target and determine its spatial position [3]. In order to achieve the best effects of detection, the working parameters of radar, such as working frequency and waveform types, are changed with different background environment. However, nowadays, the narrow bandwidth and the single emission waveform types of radar are unable to adapt to the complicated electromagnetic environment, which greatly reduces the survivability of radar and the scope of application [4]. In order to improve the ability of radar target detection in the complex electromagnetic environment, the software radio technology can be brought, and the behavior of hardware can be defined by software, thus, the working frequency, working bandwidth and waveform types can be controlled flexibly.

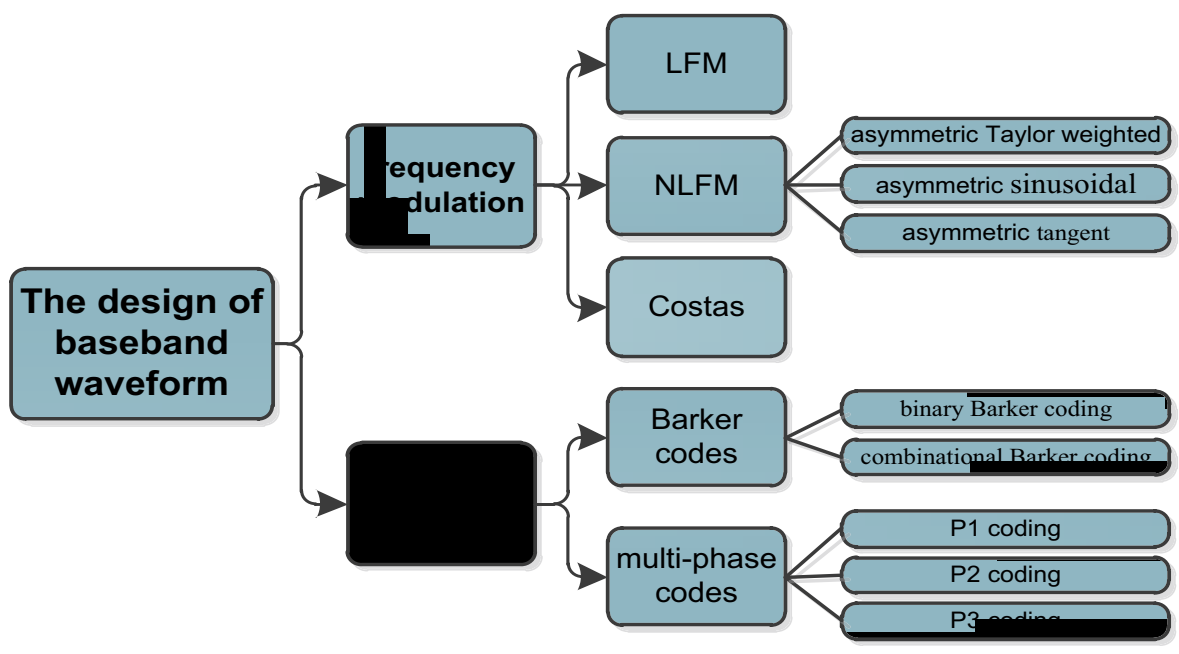

Figure 1. Design of baseband waveform

This is an Open Access article distributed under the terms of the Creative Commons Attribution License 4.0, which permits unrestricted use, distribution, and reproduction in any medium, provided the original work is properly cited. 


\section{MATEC Web of Conferences}

\section{DESIGNATION}

As shown in Figure 1, this paper introduces the design of different baseband waveform types in the pulse width through modulating the signal frequency and phase. As for the signal frequency modulation, there are linear frequency modulation (LFM), nonlinear frequency modulation (NLFM) and Costas waveform; and as for the signal phase modulation, there are Barker coding and multi-phase coded waveforms Among them, the NLFM waveform includes asymmetric Taylor weighted, sinusoidal and tangent waveform; the Barker coding waveform includes binary Barker coding and combinational Barker coding waveform; and the multi-phase coded waveform includes P1 coding, P2 coding and P3 coding waveform.

\subsection{Signal frequency modulation}

For the frequency modulation of baseband waveform, if the center frequency $f_{0}=0$, then, the LFM, NLFM and Costas waveform[5]can be expressed as the following formula(1):

$$
\begin{aligned}
f(t) & =\left\{\begin{array}{l}
\frac{B}{\tau} t \\
B\left(\frac{t}{\tau}+\sum_{n=1}^{7} K_{n} \sin \left(\frac{2 \pi n t}{\tau}\right)\right) \\
B \tan (2 \beta t / \tau) /(2 \tan \beta) \\
M-1 \\
\Delta f \sum_{i=0}^{M-1} c_{M}(i)\left[u\left(t+\tau / 2-i \tau_{c}\right)-u\left(t+\tau / 2-i \tau_{c}-\tau_{c}\right)\right]
\end{array}\right. \\
& \frac{t}{\tau}=\frac{f(t)}{B}+\frac{k}{2 \pi} \sin (2 \pi f(t) / B)
\end{aligned}
$$

The formulas (1-1) (1-5) are respectively LFM, asymmetric Taylor weighted NLFM, asymmetric tangent NLFM, Costas and asymmetric sinusoidal NLFM waveform. As for the parameters, $t \in[-\tau / 2, \tau / 2], \tau$ is the pulse width, $B$ as the sweep width; and $K_{n}$ is the Taylor weights; and $\beta=\tan ^{-1} \alpha, \alpha \in[0,+\infty], \quad \alpha$ is the control factor of side lobe level in time; and $\Delta f$ is the step frequency, $M$ as the step number, ${ }^{c} M$ as a finite set of $1 \sim M$ integers, ${ }^{\tau}=\tau / M, u(t)$ as the step function; and ${ }^{k}$ is the control factor of side lobe level in time.

According to the relationship of frequency and phase $f(t)=\frac{1}{2 \pi} \frac{d}{d t} \psi(t)$, the relationships between phase and time can be expressed as the following formula (2):

$$
\psi(t)-\psi(-\tau / 2)=2 \pi \int_{-\tau / 2}^{t} f(s) d s, \quad t \in[-\tau / 2, \tau / 2](2)
$$

In the type (2), we make the initial phase $\psi(-\tau / 2)$ $=0$. And in the discrete time condition, we can obtain the integral:

$\psi(t)=\psi\left(n T_{s}-\tau / 2\right)=2 \pi T_{s} \sum_{i=0}^{n-1} f\left(i T_{s}-\tau / 2\right) \quad, \quad n=0,1,2 \ldots, N-1$

In the type (3), $T_{s}$ is the sampling interval of time, ${ }^{N}$ as the number of sampling points, and $N=\tau / T_{\mathrm{s}}$.

Therefore, the baseband transmit waveform of I/Q channels is respectively shown as follows:

$\left\{\begin{array}{l}a_{I}(t)=A_{I} \cos \psi(t) \\ a_{Q}(t)=A_{Q} \sin \psi(t)\end{array}\right.$

Set ${ }^{A} I=1,{ }^{A} Q=1$, and other parameter sets are shown in Table 1 :

Table 1. Waveform parameters based on the type (1-1) (1-5)

\begin{tabular}{|l|l|l|l|l|l|l|l|l|}
\hline$f_{s}(1)$ & $\tau$ & $B$ & $K_{n}$ & $\alpha$ & $\Delta f$ & $M$ & $c_{n}$ & $k$ \\
\hline $80 \mathrm{MHz}$ & $5 u s$ & $20 \mathrm{MHz}$ & $(2)$ & 2.5 & $10 \mathrm{MHz}$ & 8 & $(3)$ & 0.7 \\
\hline
\end{tabular}

(1) When $f$ as a sampling rate, $f_{s}=1 / T_{s}$;

(2) $K_{n}=[-0.1145,0.0396,-0.0202,0.0118,-0.0082,0.0055,-0.0040][5]$

(3) $c_{M}=[4,3,6,5,2,0,7,1]$.

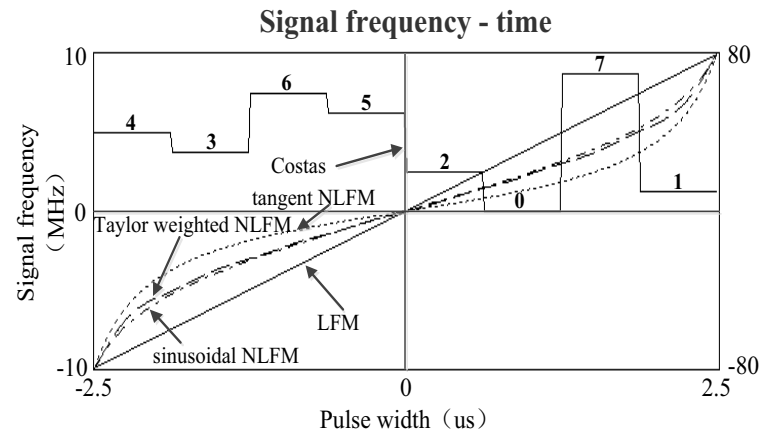

(a)

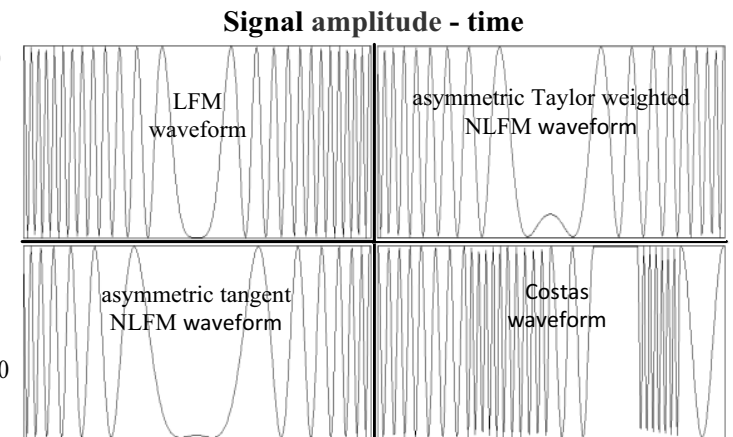

(b)

Figure 2. Diagrams of the relationship between signal frequency and time and the relationship between signal amplitude and time 
Table 2. Waveform parameters based on the type (5-1) (5-5)

\begin{tabular}{|l|l|l|l|l|l|}
\hline$f_{s}$ & $\tau$ & $N$ & $B_{i}$ & $B_{m n}$ & $M$ \\
\hline $80 \mathrm{MHz}$ & $5 u s$ & 64 & $B_{13}(1)$ & $B_{54}(2)$ & 8 \\
\hline (1) $B_{13}=[1,1,1,1,1,0,0,1,1,0,1,0,1] ;$ &
\end{tabular}

(1) $B_{13}=[1,1,1,1,1,0,0,1,1,0,1,0,1]$
(2) $B_{54}=[1,1,1,0,1,1,1,1,0,1,0,0,0,1,0,1,1,1,0,1]$.

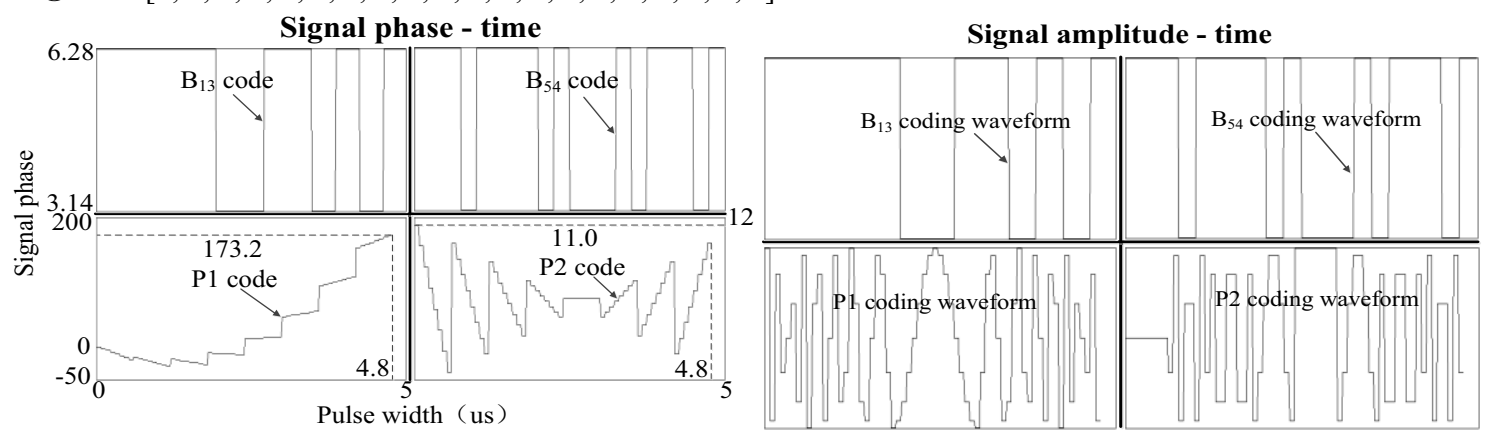

Figure 3. Diagrams of the relationship between signal phase and time and the relationship between signal amplitude and time

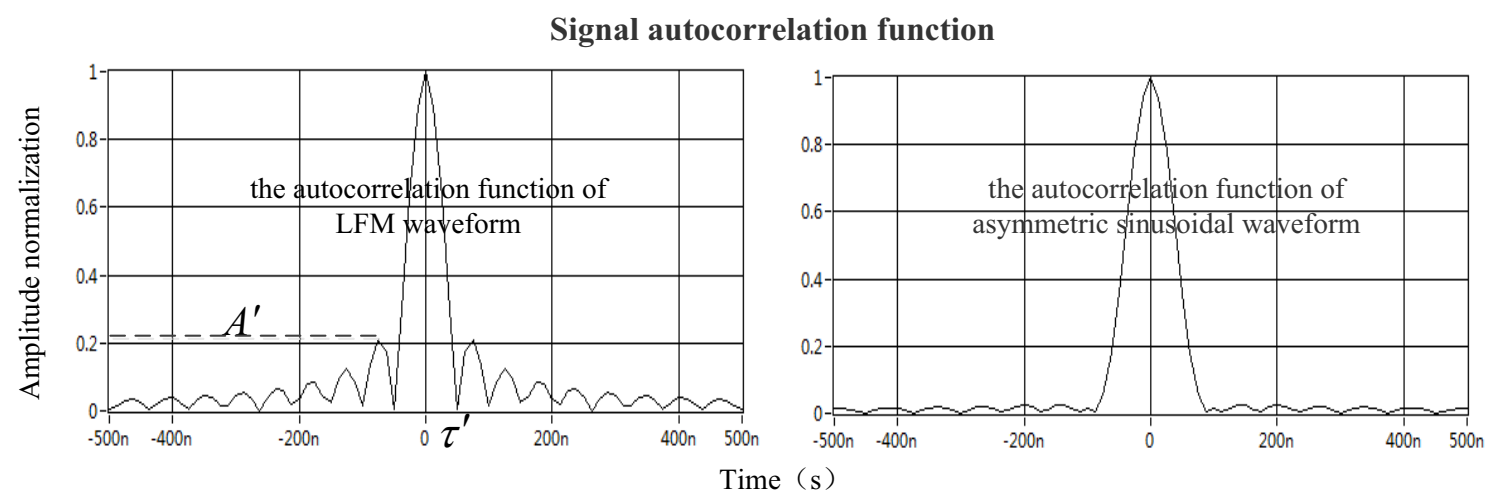

Figure 4. Autocorrelation function of NLFM and asymmetric sinusoidal waveform

Diagram the relationship between signal frequency and time based on the types (1-1) (1-5), and the relationship between part signal amplitude and time is shown in Figure 2:

Figure 2(a) is the diagram of the relationship between signal frequency and time based on the types $(1-1) \sim(1-5)$, in which the LFM, asymmetric Taylor weighted NLFM, tangent NLFM and sinusoidal NLFM waveform refer to the left vertical axis, and the Costas waveform refers to the right vertical axis. Figure 2(b) includes LFM waveform, asymmetric Taylor weighted NLFM waveform, asymmetric tangent NLFM waveform and Costas waveform.

\subsection{Signal phase modulation.}

For the phase modulation of baseband waveform, the Barker coding waveform and the multi-phase coded waveform [6] can be expressed as the following formula(5):

$$
\psi(t)=\left\{\begin{array}{l}
\pi \sum_{j=0}^{N-1}\left[B_{i}(j)+1\right][u(t-j \tau / N)-u(t-j \tau / N-\tau / N)] \\
\pi \sum_{j=0}^{N-1}\left[B_{m n}(j)+1\right][u(t-j \tau / N)-u(t-j \tau / N-\tau / N)] \\
\frac{-\pi}{M} \sum_{j=0}^{M-1} \sum_{i=0}^{M-1}[M-(2 j+1)](j M+i)[u(t-i \tau / N-j M \tau / N)-u(t-i \tau / N-j M \tau / N-\tau / N)](5-3) \\
\frac{\pi}{2 M} \sum_{j=0}^{M-1} \sum_{i=0}^{N-1}[M-(2 i+1)](M-2 j)[u(t-i \tau / N-j M \tau / N)-u(t-i \tau / N-j M \tau / N-\tau / N)](5-4) \\
\frac{\pi}{N} \sum_{n=0}^{N-1} n^{2}[u(t-n \tau / N)-u(t-n \tau / N-\tau / N)]
\end{array}\right.
$$

(5)

In the formula (5), $t \in[0, \tau], \tau$ is the pulse width, ${ }^{N}$ as the symbol length. Among them, the type (5-1) expresses the relationship between the phase of binary Barker coding waveform and time, $N=i$ ( $i$ values $2,3,4,5,7,11,13)$, and the binary Barker codes $B_{i}=$ $\{[11],[10],[110],[1101],[1110],[11101],[1110010],[11$ $100010010],[1111100110101]\}[6] ;$ and the type (5-2) relates the phase of combinational Barker coding waveforms to the time, $N=m n$ ( $m, n$ value 


\section{MATEC Web of Conferences}

$2,3,4,5,7,11,13)$. The combinational Barker codes ${ }^{B_{m n}}$ is that $B_{m}$ repeats $n$ times and correspondingly multiplies with $B_{n}$ in each repetition, which meets the rules: The same number of multiplication is 1 , and the different number of multiplication is 0 ; the type (5-3) and (5-4) are respectively the relational expression for the phase of $\mathrm{P} 1$ coding and $\mathrm{P} 2$ coding waveform with time, and ${ }^{N=M^{2}}, M$ is the order number of Frank code matrix; and the type (5-5) refers to the relationship between $\mathrm{P} 3$ coding phase and time. According to the formula (4) and $\psi(t)=\psi\left(n T_{S}\right)$, $n=0,1, \ldots, f_{S}^{\tau}$, set $A_{I}=1, A_{Q}=1$ and other parameters are shown in Table 2 .

Diagram the relationship between part signal phase and time, and the relationship between part signal amplitude and time based on the types $(5-1) \sim(5-5)$ is shown in Figure 3.

Figure 3 maps the relationship between signal phase and time, and the relationship between signal amplitude and time, which both are based on binary Barker $B_{13}$ coding waveform, combinational Barker $B_{54}$ coding waveform, multi-phase P2 coding waveform and multi-phase P2 coding waveform. At the left and right below in the Figure 3(a), the signal launch time is slightly less than the pulse width $\tau$ due to the emissive sampling points $f_{\mathrm{c}} \tau / N \approx 6$ at the launch time of code element in the condition of sampling rate $f_{\mathrm{s}}$. And the total emission points are $6 \times 64=384$, so the total launch time is $\tau^{\prime}=\tau 384 /\left(f_{s} \tau\right)=4.8<5(u s)$.

\subsection{Comparison of waveform performance}

There are ten waveforms based on the formula (1) and the formula (5). According to the signal autocorrelation function of amplitude normalization, we can give a comparison of the signal side lobe level in time, the peak side lobe ratio, the Rayleigh resolution in time, bandwidth and distance resolution. The autocorrelation function [7] is defined as follows:

$$
R_{a}(t)=\int_{-\infty}^{+\infty} a(s+t) a^{*}(s) d s=\int_{-\tau / 2}^{\tau / 2} a(s+t) a^{*}(s) d s \quad, \quad t \in[-\tau, \tau](6)
$$

If the sampling interval in time is ${ }^{T_{S}}$, then, in the pulse width, the sampling points are $N=\tau / T_{S}$, and $a(t)=a\left(n T_{\mathrm{s}}\right)$. Thus, we can obtain the autocorrelation function based on discrete time as follows:

$$
R_{a}\left(n T_{S}\right)=T_{S} \sum_{m=0}^{N-1} a\left(n T_{S}+m T_{S}\right) a^{*}\left(m T_{S}\right) \quad, \quad n=-N, \ldots 0,1, \ldots, N \text { (7) }
$$

Finally, we can obtain the signal autocorrelation function of amplitude normalization as follows:

$$
R_{a}\left(n T_{S}\right)=R_{a}\left(n T_{S}\right) / R_{a}(0)=\sum_{m=0}^{N-1} a\left(n T_{S}+m T_{S}\right) a^{*}\left(m T_{S}\right) / \sum_{m=0}^{N-1}\left|a\left(m T_{S}\right)\right|^{2}
$$

In the condition of the parameters in Table 1, the autocorrelation function of NLFM and asymmetric sinusoidal waveform are shown in Figure 4:

The peak side lobe level in time $A^{\prime}$ and the Rayleigh resolution in time $\tau^{\prime}$ can be obtained from the graphs of signal autocorrelation functions, so the peak side lobe ratio is $P S L R=-20 \log A^{\prime}$, the bandwidth is $1 / \tau^{\prime}$, and the distance resolution is $\Delta R=c \tau^{\prime} / 2$. In the condition of the parameters in Table 1 and Table 2, the performances of ten waveforms list are shown in the Table 3.

For the pulse radar target detection, if the side lobe of the transmitted waveform is too high, then the side lobe of the received signal is possible to flood the real target and causes undetectability; or it is mistaken for target and causes the error detection. Therefore, the peak side lobe waveform ratio is an important index to evaluate the quality of the waveform. However, due to the energy conservation of the signal, if the signal side lobe reduces, then more energy will be concentrated on the main lobe, and cause the main lobe broadening which makes the Rayleigh resolution in time larger and brings the distance resolution decreasing[8]. Therefore, the design of radar waveform must weigh the peak side lobe ratio and the distance resolution.

For the ten waveforms in Table 3, LFM wave-form has the best distance resolution $\Delta R=7.5 \mathrm{~m}$. However, the PSLR is minimum with $13.5 d B$ only; and the asymmetric Taylor weighted NLFM waveform has maximum PSLR which is up to $34.4 d B$, and its distance resolution is relatively good which is up to $13.1 \mathrm{~m}$. Besides, the asymmetric sinusoidal NLFM waveform and the multi-phase P3 coding waveform are also well designed waveforms. Although the PSLR is not particularly high, which are respectively $31.7 \mathrm{~dB}$ and $24.3 d B$, the distance resolution can be up to $13.1 \mathrm{~m}$ and $11.3 \mathrm{~m}$.

Table 3. Comparison of waveform performance

\begin{tabular}{|l|l|l|l|l|l|}
\hline & $A^{\prime}$ & $P S L R(d B)$ & $\tau^{\prime}(n s)$ & $1 / \tau^{\prime}(\mathrm{MHz})$ & $\Delta R(m)$ \\
\hline LFM & 0.211 & 13.5 & 50 & 20.0 & 7.5 \\
\hline asymmetric Taylor weighted & 0.019 & 34.4 & 87 & 11.5 & 13.1 \\
\hline asymmetric tangent & 0.030 & 30.5 & 240 & 4.2 & 36.0 \\
\hline asymmetric sinusoidal & 0.026 & 31.7 & 87 & 11.5 & 13.1 \\
\hline Costas & 0.174 & 15.2 & 87 & 11.5 & 13.1 \\
\hline binary Barker $B_{13}$ code & 0.143 & 16.9 & 385 & 2.6 & 57.8 \\
\hline combinational Barker $B_{54}$ code & 0.250 & 12.0 & 250 & 4.0 & 37.5 \\
\hline multi-phase P1 code & 0.041 & 27.7 & 100 & 10.0 & 15.0 \\
\hline multi-phase P2 code & 0.079 & 22.0 & 100 & 10.0 & 15.0 \\
\hline multi-phase P3 code & 0.061 & 24.3 & 75 & 13.3 & 11.3 \\
\hline
\end{tabular}


ICETA 2015

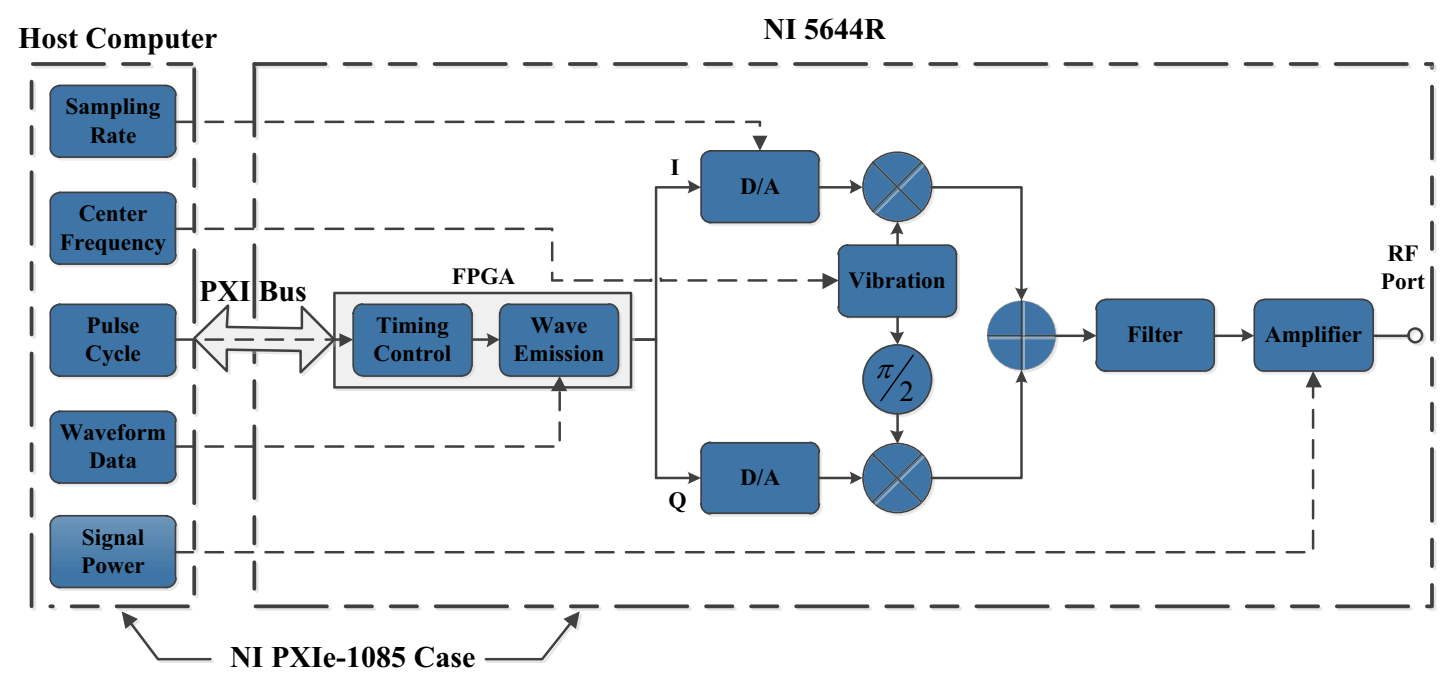

Figure 5. Schematic diagram of wave emission based on NI PXIe-5644R
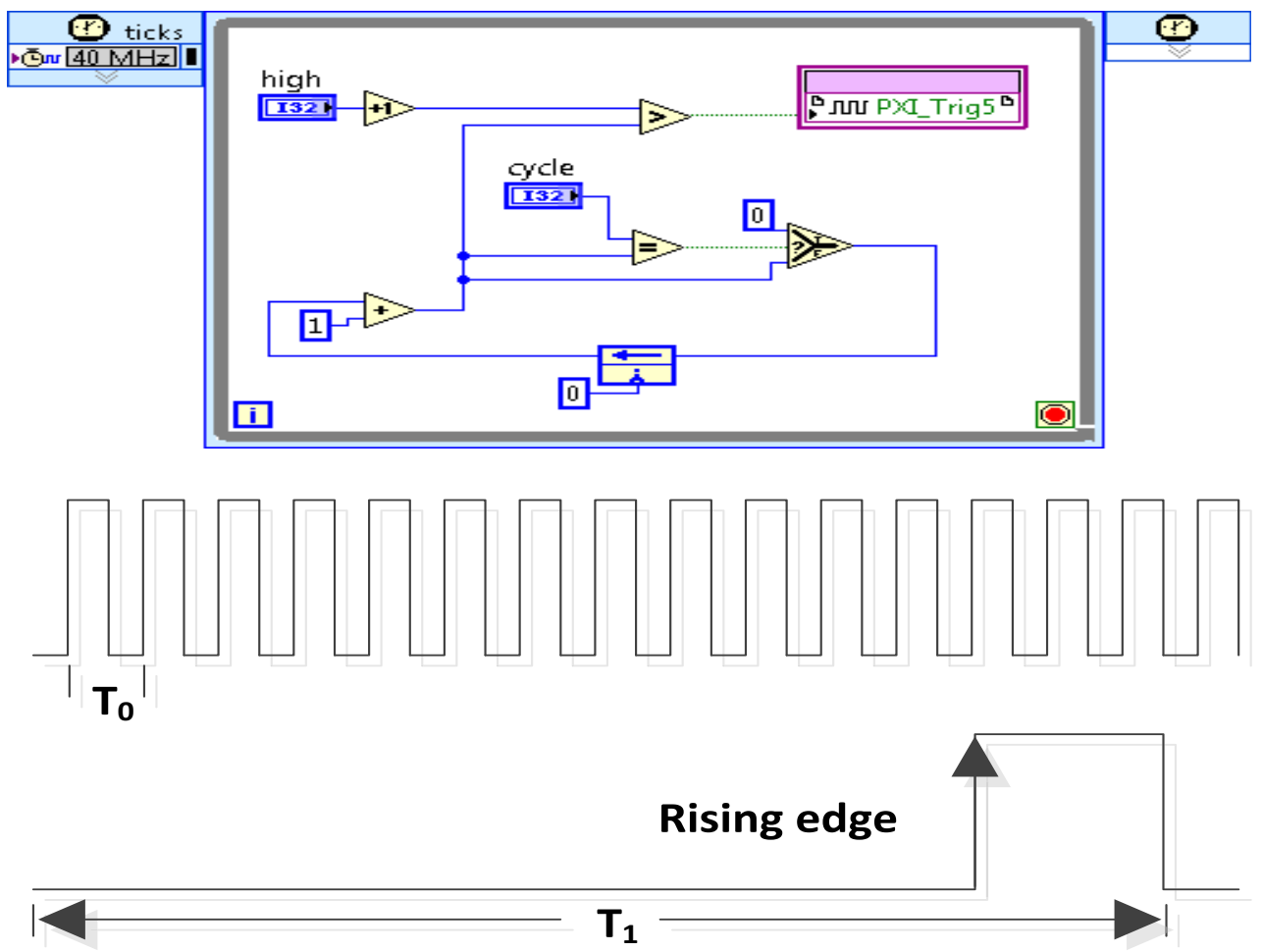

(a). Timing control procedures 
MATEC Web of Conferences

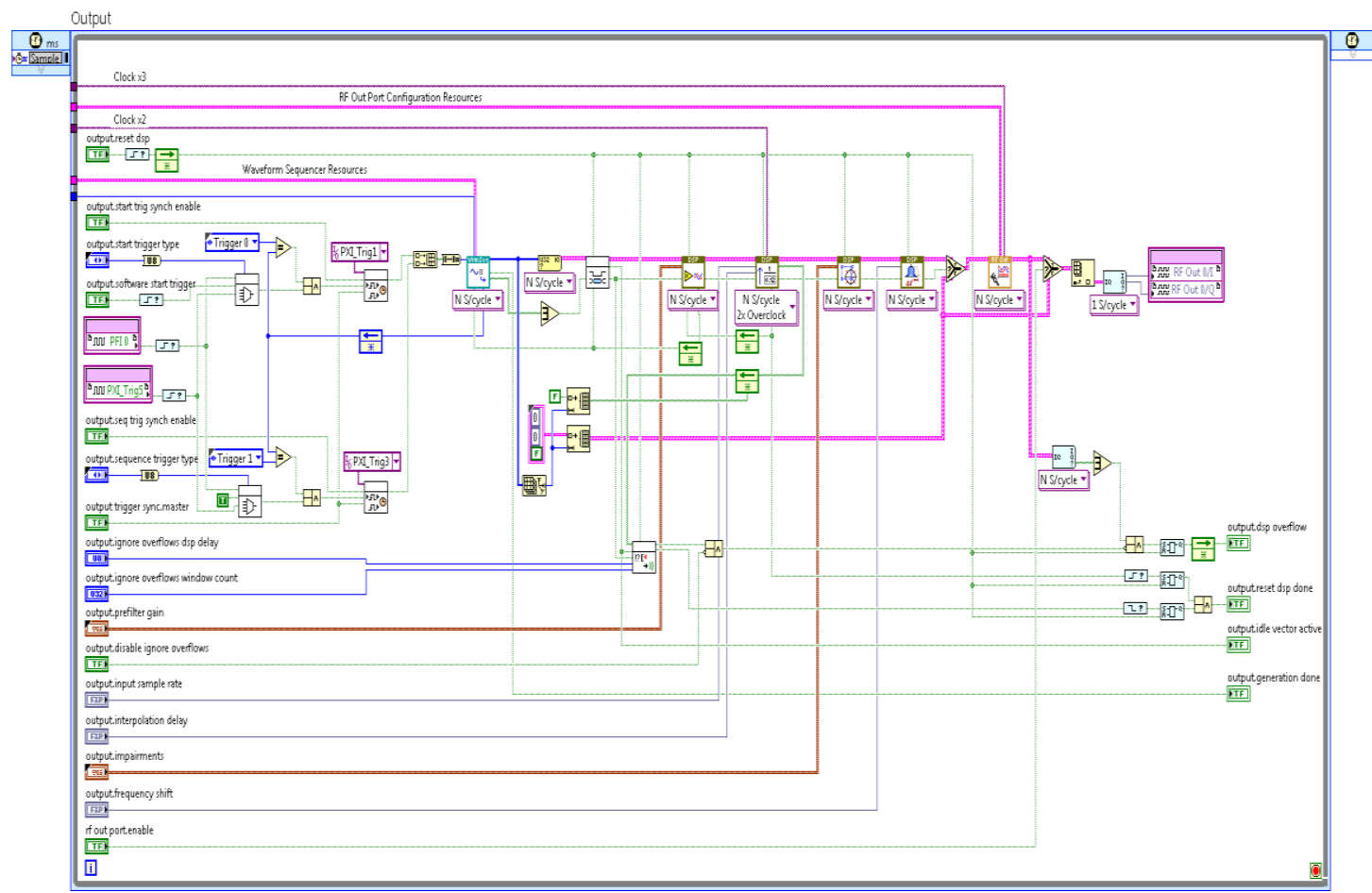

(b). Waveform launch procedures

Figure 6. Timing control procedures and waveform launch procedures

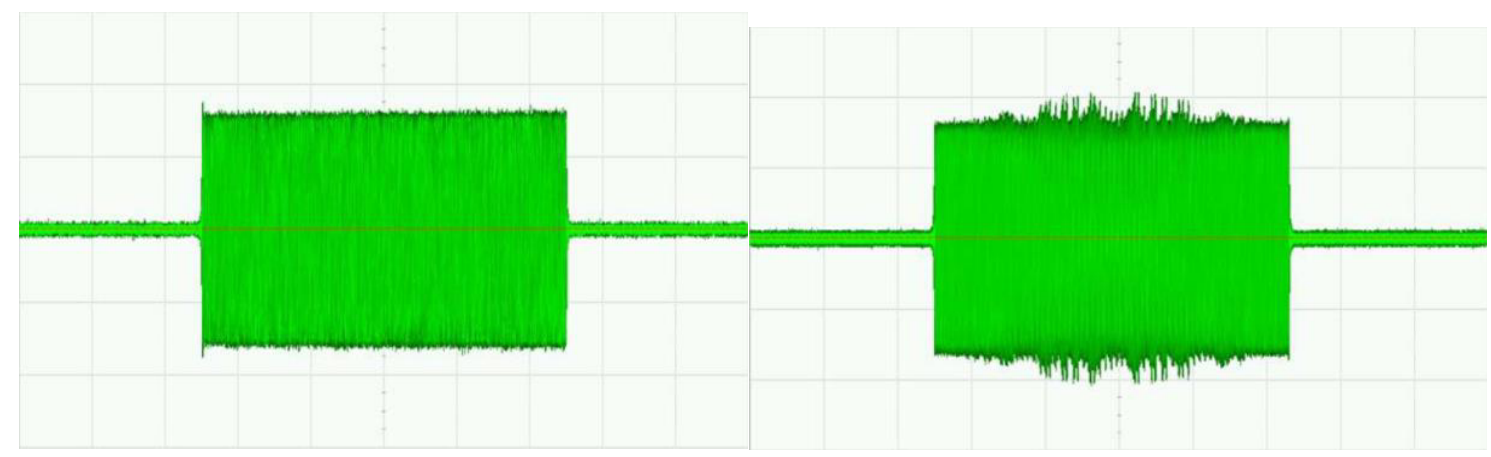

(a). Asymmetric sinusoidal NLFM waveform

(b). Multi-phase P3 coding waveform

Figure 7. RF signals

\section{REALIZATION}

Implementation of the pulse radar waveform is based on the software radio platform NI PXIe-5644R, and its working principle is shown in Figure 5. The host computer and NI 5644R are set up in the NI PXIe-1085 case, and NI 5644R includes FPGA programming module and RF module, and the PXI bus is used to exchange data between the host computer and FPGA. We change the sampling rate, center frequency, pules cycle, waveform data, signal power and other parameters in the host computer, which transfers instructions to the FPGA by PXI bus, then, the FPGA controls the RF module, such as D/A converter, vibration, filter and amplifier, and finally achieves the wave emission. 


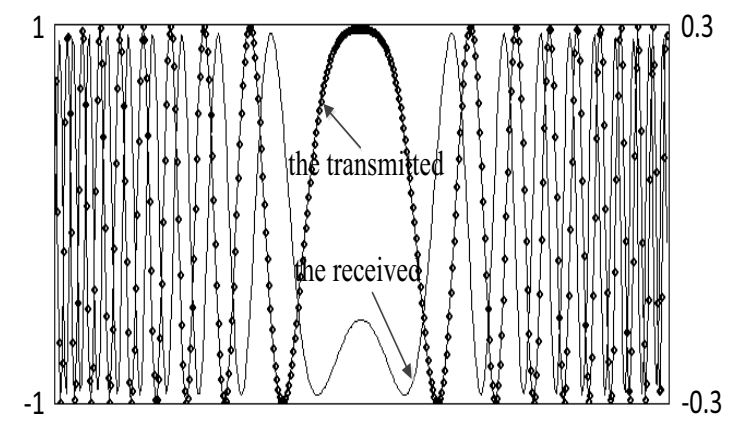

(a): asymmetric sinusoidal NLFM waveform

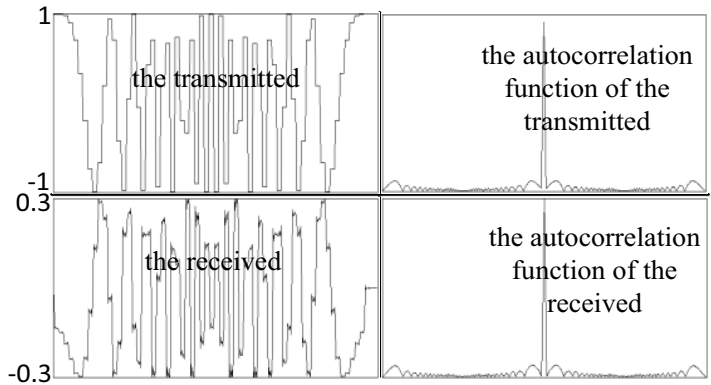

(b): multi-phase P3 coding waveform

Figure.8: the comparison of transceiver baseband waveform

To periodically launch the arbitrary waveform, we design the timing control procedures and the waveform launch procedures mainly in the FPGA module as shown in Figure 6 . And for the timing control procedures in Figure 6(a), the board clock signal frequency which is the period of $40 \mathrm{MHz}\left(T_{0}\right.$ $=25 \mathrm{~ns}$ ) is divided into the radar pulse repetition cycle $T_{1}$; and for the waveform launch procedures in Figure 6(b), the waveform data from the host computer is read into the FPGA card at the "Rising edge" triggering, and then, the RF port launches the signal out from the I/Q channels after the digital signal processing, such as the digital gain, fractional interpolation, signal frequency shift and the digital correction.

\section{TEST AND VERIFICATION}

Based on the NI PXIe-5644R, we set the sampling rate $f_{\mathrm{s}}=80 \mathrm{MHz}$, the center frequency $f_{0}=3 \mathrm{GHz}$, the pulse cycle $T_{1}=1 \mathrm{~ms}$, and the signal power $P=0 \mathrm{dBm}$. We get access to the oscilloscope at the RF port, and then we test the ten waveforms in the Table 3 . Among them, the RF signals of the asymmetric sinusoidal NLFM waveform and the multi-phase P3 coding waveform are shown in Figure 7.

Then, we use the RF receiving port of NI 5644R, and set the same parameters. After that, we demodulate and sample for each signal. By comparing the received baseband signal with the transmitted baseband signal, we verify ten waveforms in the Table 3 . Among them, the transceiver baseband signals of asymmetric sinusoidal NLFM waveform and multi-phase P3 coding waveform are shown in Figure 8.

The comparison of the transceiver baseband of the asymmetric sinusoidal NLFM waveform is in the Figure 8(a), the amplitude of the transmitted refers to the left vertical axis, and the amplitude of the received refers to the right vertical axis. Meanwhile, the Figure 8 (b) is about the multi-phase P3 coding waveform, and there are four pictures, which are respectively the transmitted P3 coding baseband waveform, the autocorrelation function of the transmitted, the received P3 coding baseband waveform and the autocorrelation function of the received.
According to the Figure 8, compared with the transmitted baseband signal, there are some phase differences and amplitude attenuations existing in the received baseband signal. That is because the signal phase will change $2 \pi$ at the signal transmission of each $\lambda$, and the signal transmission will bring about the power attenuation and cause the amplitude attenuation.

\section{SUMMARY}

In this paper, the authors design LFM waveform, NLFM waveform, Costas waveform, Barker coding waveform, multi-phase coding waveform and other ten kinds of commonly-used pulse radar baseband waveform based on the signal frequency and the phase modulation, and give a comparison of the peak side lobe ratio, the distance resolution and other parameters. Then, the authors test the RF signal based on NI PXIe-5644R and verify the correctness of the signal transceiver. The following work will carry out the related signal processing in the FPGA module, such as Pulse accumulation, Doppler processing and so on.

\section{REFERENCES}

[1] Ma Chuanqi \& Song Jinhua.2008. The research of software radio. The Science Education Article Collects, (10): 270-271.

[2] Li Hui, Fang Tianxiang \& Jiang Jun. 2006. The application of software radio to radar signal process. Tactical Missile Technology (1):92-96.

[3] Shi Yanbin \& Duan Minzhe.2006. Research on software radar technology. Electronic Engineer, 32(1): 1-4.

[4] Wang Qin, Wan Xianrong \& Yang Zijie. 2009. Software defined frequency synthesizer for multi-frequency HF surface wave radar. Systems Engineering and Electroics, 31(5): 1060-1063.

[5] Merrill 1. Skolnik. 2010. Radar Handbook. Beijing: Publishing House of Electronics Industry. 


\section{MATEC Web of Conferences}

[6] Mark A. Richards, James A. Scheer \& William A. Holm 2010. Principles of Modern Radar, Vol. I: Basic Principles. SciTech Publishing.

[7] Cheng Peiqing. 2010. Digital Signal Processing. Beijing: TsingHua University Press.

[8] Mark A. Richards. 2008. Fundamentals of Radar Signal Processing. Beijing: Publishing House of Electronics Industry. 